\title{
Facial prototype formation in children
}

\author{
DONALD INN, KATHERINE J. WALDEN, and ROBERT L. SOLSO \\ University of Nevada, Reno, Nevada
}

\begin{abstract}
In this study, memory representation, as exhibited in facial prototype formation, was examined in children between the ages of 3 and 6 . In the first part of this experiment, an Identikit was used to construct faces with the features of hair, eyes, mouth, and nose plus chin, varied systematically. A series of these faces (called exemplar faces) was presented individually to four groups of young children between the ages of 3 and 6 . Following familiarization with the faces, a recognition task was presented, which consisted of the presentation of some new faces, some previously shown faces, and the prototype face, which embodied the most frequently presented facial features. The number of false alarms on the prototype face increased from the younger to the older group, with 6-year-olds' reactions to the prototype similar to adult performance.
\end{abstract}

Recent investigations of visual perception and memory have led to the conclusion that these processes are much more complex and dynamic than the simple recording of sensory events. Studies of prototype formation are among the important contemporary experiments that have helped prompt a dynamic perceptual-memory concept. One model of concept learning that has become popular in recent years suggests that concepts are developed when sensory impressions combine in memory to form a prototype. Formation of a prototype is generally proposed to consist of the accumulation of attributes that are contained in the experienced exemplars. Thus, a prototype constitutes a summary representation and the central tendency of a range of experiences having attributes in common (Richardson \& Bhavnani, 1984).

Others suggest that the ideas of central tendency and attribute frequency are two separate models. For example, Posner and Keele (1968) developed a central tendency model which holds that a prototype is represented mathematically by a hypothetical point in multidimensional space where the means of the distances along all of the attributes of the exemplars intersect. A frequency model was proposed by Neumann (1974) in which a prototype is the pattern that incorporates the most frequently experienced features of a set of exemplars. Neumann (1977) compared the two types of prototype models and found that the characteristics of the stimuli can dictate which process a subject will use to form a prototype.

A prototype, then, can be formed on the basis of frequently experienced features. In a typical experiment in this area, subjects are presented with a series of stimuli that contain a varying number of features; this is followed by a recognition task, during which observers are asked to differentiate between new and old figures and a prototype figure. Several experiments have confirmed that adult subjects generally cannot distinguish the prototype from previously seen stimuli and frequently misidentify the pro-

Correspondence should be addressed to D. Inn, Department of Psychology, University of Nevada, Reno, NV 89557. totype as an "old" figure with greater certainty than they judge previously seen figures. These findings seem robust and range over several modalities, including geometric forms (Franks \& Bransford, 1971), kinesthetic movements (Solso \& Dollab, 1993; Solso \& Raynis, 1979), and human faces (Solso \& McCarthy, 1981).

The human face is both salient and frequently encountered by the young child. Some have argued that faces are the most important biological and social objects in our environment (Ellis, 1990) and that, for a child, the sight of his or her mother is of special significance. Infants can discriminate between familiar and unfamiliar adults in terms of voice and smell within the first weeks of life, but it is less certain when visual discrimination of faces first occurs (Bushnell, 1982).

In one study, Bushnell (1982) examined the discrimination of faces by young infants and found that discrimination is possible as early as 5 weeks of age, with critical information lying in the hair-face outline, whereas internal features were relatively unimportant until the 19th week. In yet another observation, Goren, Sarty, and Wu (1975) have reported that infants with a mean age of only 9 min tended to attend to a schematic face as contrasted with a blank head shape or a face in which the features were scrambled. Similar results have been obtained by Dziurawiec and Ellis (1986), and in a recent study by Johnson, Dziurawiec, Ellis, and Morton (1991), the basic findings of Goren et al. were reconfirmed and extended over longer periods of an infant's life.

The concept of facial prototypes in infants has been examined by Strauss (1979; see also Strauss \& Carter, 1984), who used faces constructed from a police identification kit. The results of these experiments indicate that infants of about 10 months old are able to form an abstract memory representation, or prototype, of faces, on the basis of the averaging of features.

Despite the general interest in information processing and cognition in children, and despite a reasonable corpus of information on facial identification in young people, there are few comprehensive models that relate these 
studies. One model proposed by Bruce and Young (1986; see also Bruce, 1988), however, does seem pertinent. Bruce and Young have proposed an information processing model in which facial identification is conceptualized as corresponding to a four-stage sequence. The first stage in the process is structural encoding of the raw facial image. This is followed by a fully elaborated representation of the face, and then the familiarity of the face is determined, followed by semantic information concerning the face's status, profession, and other attributes. Finally the person's name is associated with the face. But what are the processes involved in the memory of a young child as he or she experiences a variety of faces? Might the same principles of abstract memory representation that are found with adult subjects also occur somewhere during the developmental process? And, if so, at what period in the development of the child might such representations be demonstrable? These are some of the questions addressed in this paper.

\section{METHOD}

\section{Subjects}

The subjects in this study were enrolled in various day care centers, preschools, and elementary schools in the Reno/Sparks metropolitan area in western Nevada and in Lassen County, California. Of the seventyfive subjects, 18 were three-year-olds, 25 were four-year-olds, 28 were five-year-olds, and 14 were six-year-olds.

\section{Stimuli}

The faces used in this experiment were constructed from the Identikit, a face identification device used in police work that consists of a series of plastic templates, each representing a facial characteristic. In the present experiment, a prototype face was first composed and then exemplar faces were derived from it with the frequency of features of exemplar faces varied systematically. In the present experiment, only a single series of faces was used for all subjects. (Previous research by Solso and McCarthy (1981) has shown the stimulus effects are strong across many different series of faces.) The faces were constructed randomly within the constraint of the experimental manipulation mentioned above; conspicuously unusual faces were avoided. The following four facial features were varied: hair, eyes, mouth, and nose plus chin. One male and one female prototype face were constructed. From each of the prototype faces (which were considered to be $100 \%$ faces), four levels of exemplars were derived, each level representing a different degree of similarity to the prototype. The first level embodied $75 \%$ of the prototype as three out of four features were identical to the prototype. Similarly, $50 \%, 25 \%$, and $0 \%$ exemplars were constructed (see Figure 1) in which the percentage indicated the commonality of features between exemplars and prototypes. High-resolution photocopies of these faces were made and randomly collated in a booklet that contained seven faces: two $75 \%$ exemplars, three $50 \%$ exemplars, and two $25 \%$ exemplars.

\section{Procedure}

Pretest. A pretest, which consisted of a simplified version of the actual procedure, was given to each potential subject. The faces were composed similarly to the experimental faces mentioned above. The children were instructed that they were playing a type of game in which each child was presented with three faces and told, "Look at this man (or woman)." They were then given two recognition trials, which contained an "old" face (a face in the original set) and a "new" face (a face not in the original set), randomly presented, and asked, "Did I show you this picture before?" The children were encouraged to look at the entire face. Each child was given three complete pretests. Those who were unable to identify the faces correctly in at least two out of the three trials were not used in the experiment. Of the 18 three-yearold children, 12 met the criterion of successful identifications; for the

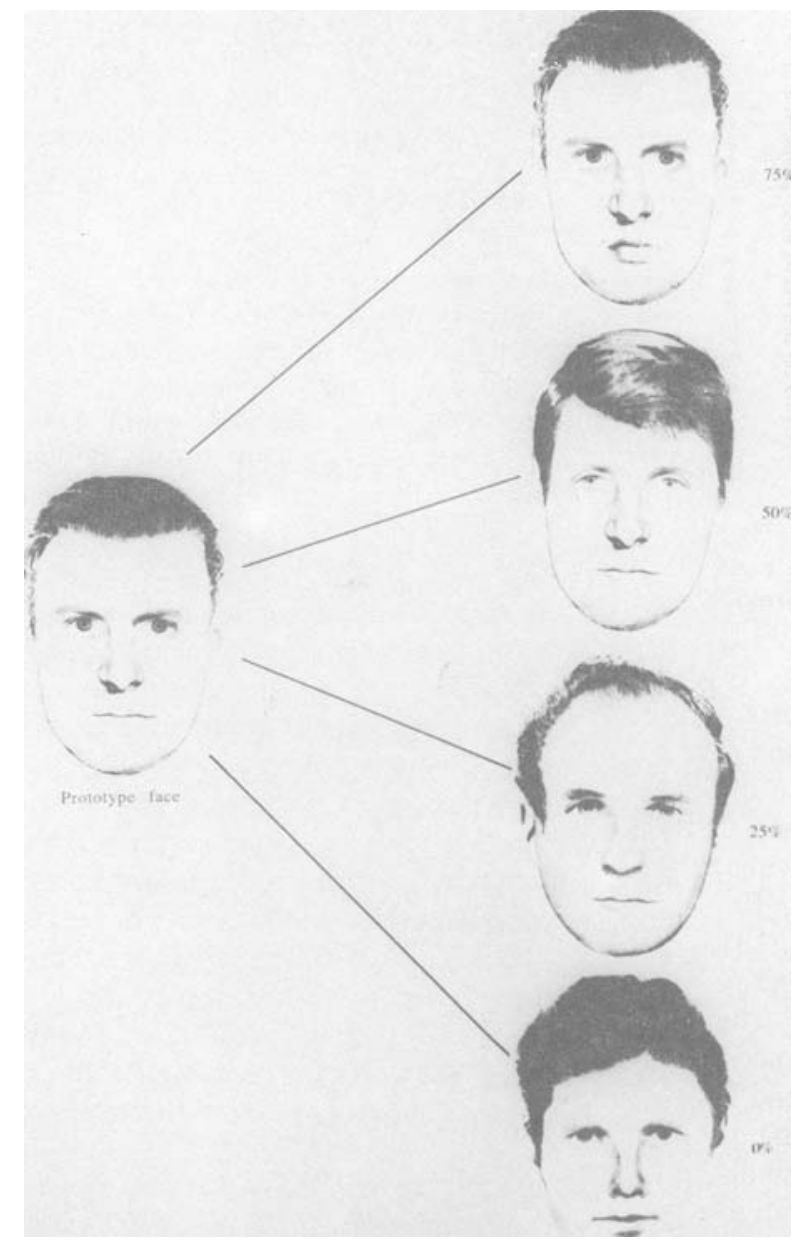

Figure 1. Examples of prototype face and exemplars used.

4-year-old group, 21 of the 25 met the criterion; and all of the 5- and 6-year-olds successfully completed the pretest.

Main experiment. In the main experiment, each child was told, "Let's try some different pictures." The experimenter first showed each face in the booklet while instructing, "Look at this man (or woman)" when each face was presented. Following this, the children were asked to view another series of faces, this time from a test booklet, and they were told, "Now look at this man (or woman). Did I show him (or her) to you before?" The test booklet contained the $100 \%$ face or prototype, which had never been seen before, a less than $100 \%$ new face, two old faces, and a $0 \%$ new face. The order was random and the rate was untimed. After the conclusion of the test condition, each child was praised with statements such as, "You won the game! You're very smart."

\section{RESULTS}

The children judged whether or not a face was an old or a new face, when the face was an old, a new, or a prototype face. Identification of an old face as "old" was considered to be a hit; as "new," a miss. Identification of a new face as "new" was considered to be a correct rejection; as "old," a false alarm. The data were analyzed by means of a pairwise $t$ test, which indicated the following: prototype versus new faces $[t(74)=2.40, p=.019]$, 




Figure 2. Percent hits on old faces and percent fales alarms on prototype and new faces.

prototype versus old faces $[t(74)=.001, p=1.000]$, and new faces versus old faces $[t(74)=2.84, p=.006]$. In the analysis of the data within each age group (Figure 2), there were no significant differences until age 6 , which yielded the following: prototype versus new faces $[t(13)=6.90, p=.0001]$, prototype versus old faces $[t(13)=1.88, p=.082]$, and new faces versus old faces $[t(13)=-4.16, p=.001]$.

The results indicate that there was a significant difference between recognition of the prototype and new faces and between new faces and old faces, but not between the prototype and old faces. In general, the data for the number of false alarms for the prototype faces and the hits for the old faces resemble each other but are both distinct from the false alarms for the new faces.

The prototype data suggest that as children get older they become more consistent in calling the prototype face an "old" face (see Figure 3). By age 6, children are performing at a level comparable to that of young adults (see Solso

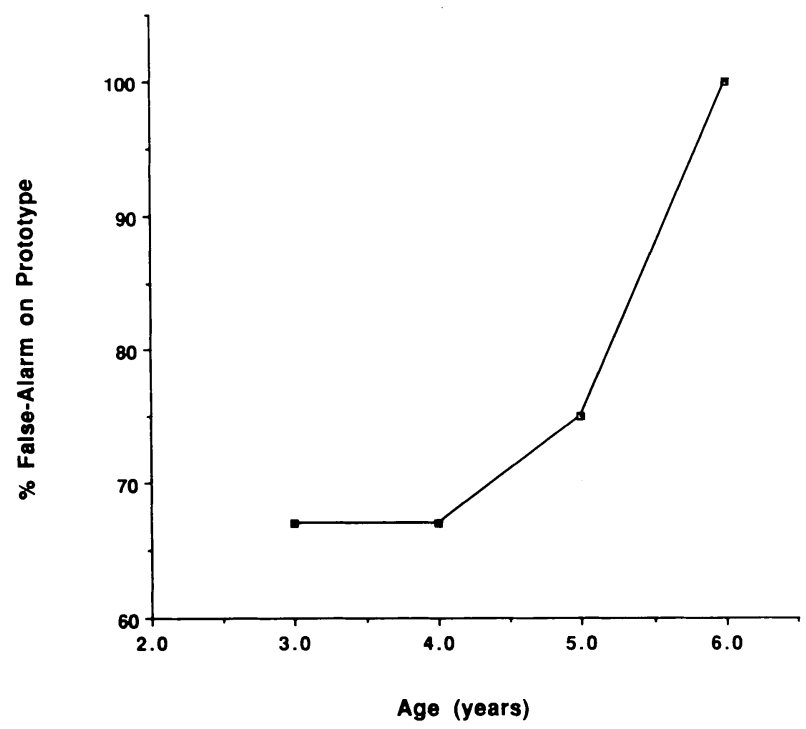

Figure 3. Percent false alarms on prototype face.
\& McCarthy, 1981). In the present study, $67 \%$ of the 3year-olds, $67 \%$ of the 4 -year-olds, $75 \%$ of the 5 -year-olds, and $100 \%$ of the 6-year-olds falsely labeled the prototype as a previously shown face. Chance performance was $50 \%$. With the data compared between age groups in an independent $t$ test, the following were found to be statistically significant: age 4 versus age $6[t(20)=3.16, p=.005]$, and age 5 versus age $6[t(27)=3.00, p=.006]$.

\section{DISCUSSION}

Dynamic theories of memory propose that the storage of information is more than a passive recording of sensory events. These theories suggest that there is an active amalgamation of sensory experiences that may take the form of a prototype, which, in the present context, is defined as a combination of the most frequently experienced features in a face. In general, the human brain stores categorically related information in terms of prototypes because of economy of storage. By storing the prototype of a class of objects, such as a prototype face, it is possible to retain, in recognition memory, the essence of a class of information such as the embodiment of most frequently experienced facial features. The preceding conclusion has been supported by many research projects with adult subjects.

It appears that by Age 6, children have developed the ability to abstract frequently experienced facial features and retain some form of that information, so that in a recognition task they tend to falsely recognize a face that embodies those features (a prototype face). The false alarm data for children at this age resemble the performance data of young adults examined in the United States as well as data from young adults in other countries. In general, this propensity was also observed in younger children. As is shown in Figure 3, the tendency for younger subjects (ages 3 and 4 ) to false alarm to the prototype face is evident. At the younger ages, the data failed to reach statistical significance, and such failure is likely due to attentional problems and subject variability rather than to cognitive deficiency in forming a prototype at this age. This inference is based not only on our observations, as presented in this paper, but also on the results presented by others using very young children (see, e.g., Strauss, 1979). Children up to the age of 5 may not be entirely able to understand the instructions given to them, they may not yet have the precise discriminatory abilities that allow them to form an internal representation for a complex visual event, or they may perform with greater variability than older children.

Our results support the attribute frequency model of prototype formation but do not address the central tendency model. Because of the nature of the task, which is based on the systematic variation of the frequency of features, in this experiment we only examined one aspect of the prototype issue. It may be that young children also employ a central tendency system in the analysis of complex visual data, and we consider such programs of research to be worthwhile. It should also be pointed out that the results do not address the possible relative saliency of the features.

In terms of an overall model of facial identification, it seems that young children operate well at the first two levels of the process as suggested by Bruce (1988; Bruce \& Young, 1986). Such a conclusion does not exclude the possibility of the later stages of facial identification (e.g., its semantic information and name) among young children. The current experiment did not address these issues. Nevertheless, it appears that young children do undertake some form of structural encoding of features and combine these features on the basis of the frequency with which they occur.

Finally, we observed a slight tendency for 4- and 5-year-old girls to false alarm more often to the prototype than boys of the same age. Although these data did not reach statistical significance, they are mentioned here as a possible area of subsequent investigation. This difference disappeared at Age 6, when all children false alarmed to the prototype, and, if gender differences exist, they would likely be obscured by ceiling effects at this level. Young girls tend to develop faster than young boys in many ways. Should such observations prove to be reliable, then perhaps we can include memory and the ability to embody common features into a prototype as ways in which boys and girls differ in early cognitive development. 


\section{REFERENCES}

BRUCE, V. (1988). Recognizing faces. Hillsdale, NJ: Erlbaum.

BRUCE, V., \& Young, A. (1986). Understanding face recognition. British Journal of Psychology, 77, 305-327.

BUSHNELL, I. W. R. (1982). Discrimination of faces by young infants. Journal of Experimental Child Psychology, 33, 298-308.

Dziurawiec, S., \& Ellis, H. D. (1986). Neonates; attention to facelike stimuli: Goren, Sarty, and Wu revisited. Paper presented to the annual conference of the British Psychological Society, University of Exeter.

ElLIS, H. D. (1990). Developmental trends in face recognition. The Psychologist: Bulletin of the British Psychological Society, 3, 114-119.

FranKS, J. J., \& BRANSFORD, J. D. (1971). Abstraction of visual patterns. Journal of Experimental Psychology, 90, 65-74.

Goren, C. C., SarTY, M., \& WU, P. Y. K. (1975). Visual following and pattern discrimination of face-like stimuli by newborn infants. Pediatrics, 56, 544-549.

Johnson, M. H., Dziurawiec, S., Ellis, H., \& Morton, J. (1991). Newborns' preferential tracking of face-like stimuli and its subsequent decline. Cognition, 40, 1-9.

Neumann, P. G. (1974). An attribute frequency model for the abstraction of prototypes. Memory \& Cognition, 2, 241-248.

NeumanN, P. G. (1977). Visual prototype formation with discontinu- ous representation of dimensions of variability. Memory \& Cognition, 5, 187-197.

Posner, M. I., \& KeEle, S. W. (1968). On the genesis of abstract ideas. Journal of Experimental Psychology, 77, 353-363.

Richardson, K., \& BhavNANI, K. K. (1984). How a concept is formed: Prototype or contingency abstraction? British Journal of Psychology, 75, 507-519.

Solso, R. L., \& Dollab, P. (1993). Prototype formation among professional dancers. Manuscript submitted for publication.

Solso, R. L., \& McCARTHY, J. E. (1981). Prototype formation of faces: A case of pseudo-memory. British Journal of Psychology, 72, 499-503.

Solso, R. L., \& RAYNIS, S. A. (1979). Prototype formation from imaged, kinesthetically, and visually presented geometric figures. Journal of Experimental Psychology: Human Perception \& Performance, 5, 701-712.

Strauss, M. S. (1979). Abstraction of prototypical information by adults and 10-month-old infants. Journal of Experimental Psychology: Human Learning \& Memory, 5, 618-663.

Strauss, M. S., \& CARTER, P. N. (1984). Infant memory: Limitations and future directions. In R. Kail \& N. E. Spear (Eds.), Comparative perspectives on the development of memory (pp. 317-323). Hillsdale, NJ: Erlbaum.

(Manuscript received December 12, 1992.) 